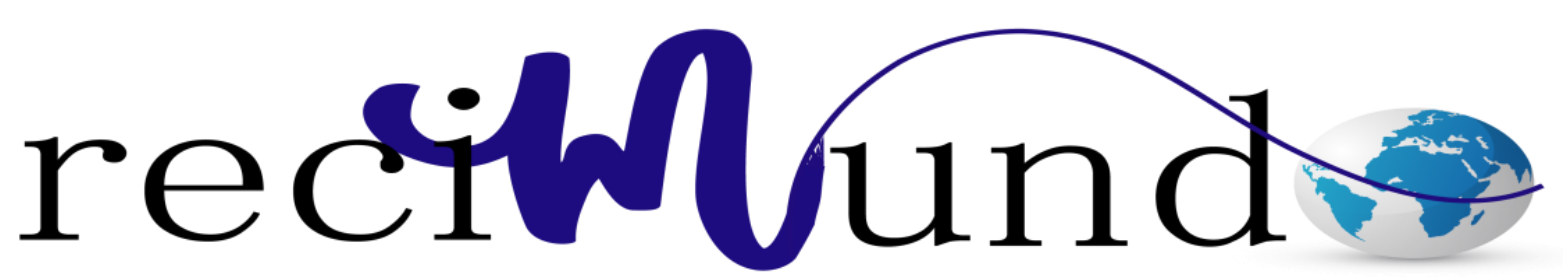

Revista Científica Mundo de la Investigación y el Conocimiento

Carlos Roberto Hernández Miticanoy a; Miguel Garcia Jiménez ${ }^{\text {b; }}$ Miguel Ángel

Aizaga Villate ${ }^{c}$

Subsidios a los hidrocarburos en Ecuador

Subsidies to hydrocarbons in Ecuador

Revista Científica Mundo de la Investigación y el Conocimiento. Vol. 3 núm. 4., diciembre, ISSN: 2588-073X, 2019, pp. 243-270

DOI: $10.26820 /$ recimundo/3.(4).diciembre.2019.243-270

URL: http://recimundo.com/index.php/es/article/view/639

Código UNESCO: 5308.01 Metodología Económica

Tipo de Investigación: Artículo de Revisión

(C) RECIMUNDO; Editorial Saberes del Conocimiento, 2019

Recibido: 15/09/2019

Aceptado: 23/11/2019

Publicado: 30/12/2019

Correspondencia: marthica12@msn.com

a. Magister en Administración Pública Mención en Gestión por Resultados; Licenciado en Ciencias de la Educación Mención Físico Matemáticas; Universidad Israel; Quito, Ecuador; rhmcarlos@ hotmail.com

b. Doctor dentro del Programa de Doctorado Hacia un Nuevo Derecho Público; Licenciado en Derecho; Universidad Israel; Quito, Ecuador: mgarcia@u.israel.edu.ec

c. Magister en Relaciones Industriales; Doctor en Ciencias Administrativas; Universidad Israel; Quito, Ecuador; maizaga@u.israel.edu.ec 


\section{Subsidios a los hidrocarburos en Ecuador}

Vol. 3, núm. 4, (2019)

Carlos Roberto Hernández Miticanoy; Miguel Garcia Jiménez; Miguel Ángel Aizaga Villate

\section{RESUMEN}

Desde su estreno como país petrolero, el Ecuador, ha subsidiado el consumo de los derivados de los Hidrocarburos, carga que ha ido creciendo anualmente, hasta convertirse en una "obligación" insostenible. El propósito de este estudio es demostrar la imperiosa necesidad de generar Políticas Públicas que permitan un manejo sostenible de los Hidrocarburos y sus derivados, en el Ecuador. Para ello se analiza la Carga Fiscal que representaron estos subsidios en el Presupuesto General del Estado durante el periodo 2009-2018, y se examina la Ley y Políticas Públicas que comprende la Administración de los Hidrocarburos. La base teórica constituye: La importancia de los Hidrocarburos en Ecuador, Los derivados de los Hidrocarburos más usados en Ecuador, Los subsidios en Ecuador, Modelos de países que no subsidian su uso y tecnologías alternativas. La investigación realizada es de tipo documental, incluye la revisión de Proformas Presupuestarias, Informes Institucionales, Ley de Hidrocarburos, fuentes bibliográficas y electrónicas. El enfoque es mixto, cuantitativo, al analizar datos numéricos, de tablas y gráficas, y; cualitativo, al analizar políticas, leyes y reglamentos del sector Hidrocarburífero del país. Los resultados muestran que el Monto destinado a subsidiar el uso de los derivados del Petróleo en el Ecuador durante el período 2009 - 2018, representa el 9,35 \% del Presupuesto General del Estado. Esto origina un alto costo de oportunidad para el Estado, evidenciado en la Desinversión, Crecimiento de la deuda Pública, Falta de Liquidez para Amortizar la misma Deuda y finalmente una ineficaz Redistribución de la Riqueza. También se encontró una excelente Constitución que no se cumple en la práctica y una Ley de Hidrocarburos fuera de contexto. Figura entre las recomendaciones: La urgencia de eliminar los subsidios a los derivados de los Hidrocarburos en pro de generar un desarrollo sostenible para el país.

Palabras Clave: Presupuesto General del Estado, Subsidios; Derivados de los Hidrocarburos; Políticas Públicas; Desarrollo Sostenible; Proformas Presupuestarias. 


\begin{abstract}
From its launch as an oil-producing country Ecuador has allocated a considerable amount of its butget to subsidise the consume of hidrocarbon derivatives. A burden that increased annually, until it became an unsustainable "obligation". The aim of this study is to show the impelling need to create public politics that allow a sustainable management of hydrocarbons and its derivatives in Ecuador. Therefore the tax burden that represent the hydrocarbon subsidies in the annual general butget in the period 2009-2018 is analysed and the laws and public politics that rule the administration of hydrocarbons are examined. The theoretical basis constitutes: The importance of hydrocarbons in Ecuador, the hydrocarbons derivatives most used in Ecuador, the subsidies in Ecuador, models of countries not subsidise the use and alternative technologies. The present research is a documentary type based on the review of pro forma budgets, institutional reports, the law of hydrocarbons, bibliographic and digital sources. The approach is integrated: quantitative, analysing numeric data of charts and graphics, and qualitative, analysing politics, laws and regulations of the Ecuadorian hydrocarbon sector. The results shows that the amount meant to subsidise the use of petroleum derivatives in Ecuador in the period 2009 - 2018 represent 9,35\% of the total amount of the general national budget in the same period. This fact causes a high opportunity cost shown by the low investmen in strategic sectors, constant increase of public debt, the lack of liquidity to amortize this debt and the inefficient redistribution of wealth. It also has been found an excellent constitution which is not complied with in practice and a law of hydrocarbons out of context. Among the recommendations: The urgency to eliminate the subsidies of hydrocarbon derivatives for the sake of generating a sustainable development for the country.
\end{abstract}

Key words: General national budget; Subsidies; Hydrocarbon derivatives; Public politics; Sustainable development; Pro forma budget. 


\section{Subsidios a los hidrocarburos en Ecuador}

Vol. 3, núm. 4, (2019)

Carlos Roberto Hernández Miticanoy; Miguel Garcia Jiménez; Miguel Ángel Aizaga Villate

\section{Introducción.}

Los subsidios a los derivados de los Hidrocarburos, constituyen una carga monetaria muy grande para el Estado ecuatoriano, esto, según varias tesis de grado, análisis económicos de expertos, así como informes de instituciones no gubernamentales. En un reporte, EL Telegrafo (2018), da a conocer que: "La proforma presupuestaria de 2019 asigna un total de \$4.176,47 millones para subsidios a combustibles" (p.1), noticia que se confirma al revisar la Programación 2019-2022; en cuyos detalles se observa que, el costo de los subsidios a los hidrocarburos en el 2019, representará un 13\% del Presupuesto General del Estado (4176,47millones de dólares) (Ministerio de Econimía y Finanzas, 2019, p. 35).

Esta situación pasaría desapercibida, si el Ecuador gozara de un sistema Económico estable y de Finanzas suficientes para cubrir tal <<aparente obligación〉>, cosa que no es así, pues año tras año crece el monto de la deuda externa, como lo señala el mismo Ministro de Economía y Finanzas, Econ. Richard Martínez en una entrevista: "hay una necesidad de financiamiento por 8.000 millones de dólares, (...), a pesar de que ha existido una reducción del déficit, todavía el país requiere seguir endeudándose, este endeudamiento, (...) se debe a que el Estado se excedió en gastos" (El Universo, 2019, p. 1)..

Los subsidios a los Hidrocarburos, se incluyen en el conjunto de los denominados “excesivos gastos", estos superan el cincuenta por ciento de la necesidad de financiamiento para el 2019. Ante aquello, surge la necesidad de trabajar en propuestas y políticas, encaminadas a propiciar una administración sostenible de los Hidrocarburos en el Ecuador, cuanto por definición son recursos no renovables. 
Subsidios a los hidrocarburos en Ecuador

Vol. 3, núm. 4, (2019)

Carlos Roberto Hernández Miticanoy; Miguel Garcia Jiménez; Miguel Ángel Aizaga Villate

Otra razón radica en que, los subsidios a los derivados de los hidrocarburos han perdido enfoque y favorecen a una porción de la población que no lo necesita; como grandes industrias, propietarios de automóviles, quienes los utilizan para uso personal, negocio e incluso lujo, situación que genera injusticia social e ineficaz distribución y redistribución de la riqueza.

Según datos estimados por el MEF, al Estado le cuesta US\$ 282 millones el subsidio al gas y US\$ 357 millones el subsidio al diésel, sumando en total US\$ 638,8 millones, que representan un 2,1\% del PIB. Este monto es aproximadamente igual a la recaudación total del impuesto a la renta en el 2004. Por lo expuesto, es urgente generar acuerdos con la sociedad, que permitan la gradual disminución de estos subsidios. Esto debería ir acompañado de reformas estructurales al sector eléctrico y de hidrocarburos, que permitan reducir los costos vía aumentos en la eficiencia e incentiven la inversión (CEPAL, 2006, p. 46).

Como se puede apreciar, las recomendaciones vienen insinuándose, desde mucho antes del período en estudio. Mientras esto ocurre, el mercado automotriz crece en un 30\%, según las propias palabras de uno de sus directivos: "Genaro Baldeón, presidente de la Asociación de Empresas Automotrices de Ecuador (AEADE), manifestó; que se tiene estimado que hasta finalizar el año, se registre un crecimiento de al menos el 33\%, con relación al año pasado”. (El Teelegrafo, 2018, p.1). La pregunta ineludible viene a ser entonces: ¿Cuánto le cuesta esto al país?, y antes de alcanzar a contestarla surge otra con la misma premura: ¿Acaso este crecimiento del mercado automotriz, se debe a que al usuario no le cuesta el combustible, como al Estado? 


\section{Subsidios a los hidrocarburos en Ecuador}

Vol. 3, núm. 4, (2019)

Carlos Roberto Hernández Miticanoy; Miguel Garcia Jiménez; Miguel Ángel Aizaga Villate

El debate sobre la pertinencia o no de continuar subsidiando el consumo de los derivados del petróleo, debido a sus altos costos, crece en la sociedad, en base a aquello, surge la necesidad de plantear el siguiente problema de estudio:

“Es necesario generar Políticas Públicas para el sector de los Hidrocarburos y sus derivados, de modo que contribuyan al desarrollo sostenible del Ecuador"

He ahí la relevancia de este estudio, el cual, a través de un proceso técnico de revisión de su normativa y el análisis de las proformas presupuestaria anuales y cuatrianuales, procurará solventar las siguientes interrogantes: ¿Qué porcentaje del Presupuesto General del Estado representan los subsidios a los Hidrocarburos en el Ecuador?, ¿Cuánto afectan a los Ingresos y Egresos del Presupuesto General del Estado, ¿Qué refiere el marco legal actual respecto a los subsidios a los Hidrocarburos en el Ecuador?, ¿Qué Politicas Públicas son adecuadas y aplicables para el menejo sostenible de los Hidrocarburos en el Ecuador?

Para ello, se plantea como objetivo general: "Proponer Políticas Públicas que permitan un manejo sostenible de los Hidrocarburos en el Ecuador" y como Objetivos específicos:

1.- Analizar la Carga Fiscal que representan los subsidios a los Hidrocarburos en el Ecuador, mediante el estudio de sus Presupuestos Generales Anuales.

2.- Comparar los Montos destinados a subsidiar el uso de los derivados de los Hidrocarburos en el Presupuesto General del Estado, con los Montos de Ingresos, por Impuesto, Tasas y Contribuciones, Financiamiento Público, Ingresos Petroleros, y Egresos, por concepto de: Amortización de deuda, Inversión en Obra Pública, Total de Subsidios y Subsidio al gas licuado de petróleo. 
Subsidios a los hidrocarburos en Ecuador

Vol. 3, núm. 4, (2019)

Carlos Roberto Hernández Miticanoy; Miguel Garcia Jiménez; Miguel Ángel Aizaga Villate

3.- Examinar la Normativa y Políticas Públicas vigentes, para reconocer el campo legal de la Administración de los subsidios a los Hidrocarburos en el Ecuador.

4.- Proponer Políticas Públicas alternativas en pro de mejorar la administración de los Hidrocarburos y sus derivados, en el Ecuador.

\section{Contexto}

A partir del 2009, "luego de la recuperación de la crisis económica, se incrementaron los precios y la demanda del consumo de gasolinas y diésel reanudando la preocupación mundial por la tendencia al consumo de Hidrocarburos y sus repercusiones en el medio ambiente" (Mendoza, 2014, p. 5). Esta preocupación no excluye a Latinoamérica, principalmente a países donde se mantienen subsidios o impuestos que fomentan la demanda de estos derivados de petróleo a sabiendas de que las condiciones económicas propias e internacionales, muestran una necesidad de frenarla (Mendoza, 2014).

El Ecuador no escapa a esta realidad, lamentablemente. Aunque en los últimos años ha empezado a echar raíces la idea de eliminar los subsidios a los derivados del petróleo, y si bien es cierto a finales del 2018, el Gobierno Nacional, mediante decreto ejecutivo descongeló los precios de los derivados del Petróleo, el problema sigue intacto, pues al comparar el monto destinado a subsidiar el consumo de los combustibles en el 2018 (1707,04 millones), este se ha incrementado en un $245 \%$ para el 2019 (4176,47millones de dólares).

El debate en tanto, se lo ha puesto en el cajón del olvido, mientras para financiar los baches presupuestarios, se "pretende privatizar algunos sectores estratégicos" idea expresada por el 


\section{Subsidios a los hidrocarburos en Ecuador}

Vol. 3, núm. 4, (2019)

Carlos Roberto Hernández Miticanoy; Miguel Garcia Jiménez; Miguel Ángel Aizaga Villate

propio Carlos Pérez García, Ministro de Energía y Recursos Naturales, en una entrevista realizada y transmitida por la cadena de televisión Ecuavisa, el ocho de julio de 2019.

\section{Importancia de los Hidrocarburos en Ecuador}

Hablar de Hidrocarburos es hablar de fuentes de energía: El transporte, la generación eléctrica, la construcción de carreteras, la cocción de alimentos, son varios de sus usos en el país.

"Los hidrocarburos son una fuente importante de generación de energía para las industrias, para nuestros hogares y para el desarrollo de nuestra vida diaria. Pero no son sólo combustibles, sino que a través de procesos más avanzados se separan sus elementos y se logra su aprovechamiento a través de la industria petroquímica” (Universidad Autónoma del Estado de Hidalgo, 2019, p. 1).

En cuanto a lo económico, los recursos petroleros constituyen un porcentaje representativo en los ingresos al Presupuesto General del Estado, así; "Entre enero de 2003 y el primer trimestre de 2008, según cifras del BCE los ingresos por exportaciones petroleras del Gobierno Central sumaban US\$ 8.022 millones” (Cueva y Ortiz, 2013, p. 10) y en los años siguientes, según las Programaciones Presupuestarias Cuatrianuales, del el período (2010 - 2018), los ingresos representarían 26346 millones de dólares. (Ministerio de Economía y finanzas, 2019).

\section{Derivados de los Hidrocarburos más usados en Ecuador}

Los Hidrocarburos más usados en Ecuador según Petroecuador son: El asfalto, combustible para pesca artesanal, solvente industrial, spray oíl, fuel oíl, jet fuel, nafta base, el diésel $(1,2)$, gasolina (súper y extra), y el gas de uso doméstico (Petroecuador, EP, 2018, p. 57), de los cuales, 
Subsidios a los hidrocarburos en Ecuador

Vol. 3, núm. 4, (2019)

Carlos Roberto Hernández Miticanoy; Miguel Garcia Jiménez; Miguel Ángel Aizaga Villate

los tres últimos se utilizan principalmente para transporte urbano, de carga o personal, para producción de electricidad, industria, y la cocción de alimentos. De estos, la mayoría son importados.

"En la proforma presupuestaria de 2014, se destinaban \$ 1.860 millones para el subsidio al diésel importado, \$ 1.300 millones para gasolina importada, \$ 570 millones para gas licuado de petróleo importado (GLP) \$ 102 millones para GLP nacional, \$ 56 millones en jet fuel importado" (Fierro, 2014, p 1).

\section{Los Subsidios en Ecuador}

Los subsidios por definición son una ayuda económica para atender ciertas necesidades individuales y colectivas, se dice también que deben tener una temporalidad bien definida, para poder medir su impacto. De no ser así, estos se naturalizan y pasan a ser una carga más para el estado, sin ningún rol importante en la sociedad. Esto último ha ocurrido en el Ecuador, cuyos subsidios han ido desvirtuándose hasta convertirse en una carga permanente, creciente y desenfocada que contribuye solamente al despilfarro del patrimonio económico del país.

La política de subsidios en el país ha tenido como efecto la migración, dentro de los sectores de consumo, a tecnologías que utilizan energía subvencionada para un uso en fines ajenos a los que fueron inicialmente pensados $y$, a su vez, la fuga de combustibles por las fronteras, dada la considerable diferencia de precios con los países vecinos. Se ha determinado que los subsidios al diésel, gasolina y al gas licuado de petróleo (GLP) benefician, en su mayoría, a los grupos sociales y económicos con mayores ingresos, profundizando la desigualdad social. (Espinoza y Guayanlema, 2017, p.1). 


\section{Subsidios a los hidrocarburos en Ecuador}

Vol. 3, núm. 4, (2019)

Carlos Roberto Hernández Miticanoy; Miguel Garcia Jiménez; Miguel Ángel Aizaga Villate

Es menester mencionar que en el Ecuador, el subsidio a los combustibles no surgió con el objetivo de ayuda social, sino que surgió a partir de 1974 "debido al precio internacional del petróleo, y su alto volumen de exportación, lo que produjo un aumento sustancial de ingresos fiscales y un excedente económico en el país. Esto, en principio, permitió abastecer los requerimientos del transporte militar” (Espinoza \& Guayanlema, 2017, p. 1), luego se amplió al transporte público y al Gas Licuado de Petróleo. Además, estos obedecen a sugerencias de los grandes exportadores de crudo y sus derivados, para lo cual:

“La justificación más utilizada por los gobiernos es de hacer partícipe a la sociedad del beneficio por ser "dueños" de un recurso abundante (petróleo), que se refleje por el aumento de los subsidios o la reducción de los impuestos de las gasolinas y el diésel. (Mendoza, 2014, p. 7).

Los subsidios que se aplican en el Ecuador al 2019, aparecen en la siguiente tabla:

Tabla 1. Subsidio del Estado (USD millones)

\begin{tabular}{|c|c|}
\hline TIPO DE SUDSIDIO & $\begin{array}{c}\text { PROFORMA } \\
2019\end{array}$ \\
\hline Combustible* & \\
\hline Diésel importado & 1923,77 \\
\hline nafta importada & 1311,30 \\
\hline GLP importado & 567,50 \\
\hline GLP nacional & 124,57 \\
\hline Otros importados** & 249,33 \\
\hline $\begin{array}{c}\text { Total subsidio } \\
\text { combustibles }\end{array}$ & $\mathbf{4 1 7 6 , 4 7}$ \\
\hline Seguridad Social & 1995,01 \\
\hline Desarrollo Social & \\
\hline Bono de desarrollo & 251,22 \\
\hline
\end{tabular}




\begin{tabular}{|c|c|}
\hline humano & \\
\hline $\begin{array}{c}\text { Pensión de adultos } \\
\text { mayores }\end{array}$ & 312,51 \\
\hline $\begin{array}{c}\text { Pensión para personas con } \\
\text { discapacidad }\end{array}$ & 84,90 \\
\hline $\begin{array}{c}\text { Bono Joaquín Gallegos } \\
\text { Lara }\end{array}$ & 93,28 \\
\hline Misión ternura & 27,38 \\
\hline $\begin{array}{c}\text { Total subsidio Desarrollo } \\
\text { Social }\end{array}$ & 769,28 \\
\hline Total subsidio Agrícola & 14,72 \\
\hline Total subsidios & $\mathbf{6 9 5 5 , 4 8}$ \\
\hline
\end{tabular}

Fuente: Programación Presupuestaria Cuatrianual (PPC) 2019-2022. Elaborado por: Ministerio de Economía y Finanzas.

Modelos de países que Eliminaron los Subsidios a los derivados de los Hidrocarburos

A nivel mundial, existe una gran cantidad de países que no subsidian el uso de los derivados del petróleo, entre ellos los desarrollados, en Latinoamérica existen países como México, Brasil, Colombia en donde se han eliminado los subsidios a los derivados del petróleo. Mendoza (2014) menciona: "En Colombia se eliminaron los subsidios a las gasolinas y diésel desde 2011, los años de 2009 y 2010 fueron para el proceso de desmonte de los subsidios" (p.14).

En Brasil, país que se ha convertido en un referente para Latinoamérica, es otro país mencionado en el estudio, en él, el proceso de eliminación de subsidios ha transitado un largo camino, hasta llegar a su cometido, "Esta medida fue estudiada en una agenda política en la que, se inició, con la remoción de subsidios de los combustibles de menor interés político y social. 


\section{Subsidios a los hidrocarburos en Ecuador}

Vol. 3, núm. 4, (2019)

Carlos Roberto Hernández Miticanoy; Miguel Garcia Jiménez; Miguel Ángel Aizaga Villate

Posteriormente, se trataron los grupos más vulnerables, como, transporte e industria.” (Espinoza y Guayanlema, 2017, p.18).

Modelos de Aplicación de Fuentes Alternativas de Energía

Existen países, que ya han entrado al nuevo mundo, refiriéndonos al sentido de cambiar las fuentes de energía, para la movilización, la industria y el uso doméstico que son los puntos más sensibles del tema en estudio. Tal es el caso de la ciudad Shennzhen (China), en cuya ciudad. "Bajo una nueva ley aprobada este 2017, a partir de 2018 todos los autobuses que presten el servicio de transporte para pasajeros dentro de Shenzhen deberán ser eléctricos, por lo que queda prohibido usar autobuses de combustión interna" (Xakata, 2018, p.1). Este ejemplo lo ha seguido la empresa Saucinc de Guayaquil, con la adquisición de veinte buses de esta clase. El uso de estos buses incrementa el pasaje en 10 centavos por pasajero. También lo viene utilizando el sistema Trolebus en Quito desde el año 1995, con un costo del pasaje igual al del transporte convencional.

Otra alternativa es la que planteó el gobierno del Econ. Rafael Correa, en su afán de cambiar la matriz productiva y la sustitución de fuentes (GLP por electricidad generada por hidroenergía), cuyo proyecto, entre otras estrategias, contemplaba: "Los incentivos que el Estado entrega al ciudadano, para que opte por el cambio a tecnología de inducción son: (...) iii) subsidio exclusivo para beneficiarios del Bono de Desarrollo Humano del 100\% del costo de la cocina de inducción" (Espinoza y Guayanlema, 2017, p. 24).

\section{Métodos y materiales.}

El presente estudio corresponde a un Análisis Documental de tipo Descriptivo cuyo Diseño Metodológico es no experimental longitudinal puesto que se ha escogido intencionalmente el 
Subsidios a los hidrocarburos en Ecuador

Vol. 3, núm. 4, (2019)

Carlos Roberto Hernández Miticanoy; Miguel Garcia Jiménez; Miguel Ángel Aizaga Villate

período 2009-2018 para analizar las Proformas Presupuestarias Anuales y Cuatrianuales, a fin de interpretar esos datos y relacionarlos con los Montos destinados a subsidiar el uso de los derivados de los Hidrocarburos en el Ecuador. Esta investigación, asumió el enfoque Mixto por ser el más adecuado, pues para el caso, será necesaria la interpretación cualitativa de las fuentes bibliográficas como: La Ley de Hidrocarburos, Informes Ministeriales, Tesis referentes a Impactos Sociales de la focalización o eliminación de algunos de los subsidios, y; la interpretación cuantitativa de las variables escogidas del Presupuesto General del Estado y los Subsidios a los Hidrocarburos, que aparecen en tablas y gráficos estadísticos presentados por las Instituciones Estatales como: el Banco Central del Ecuador, Ministerio de Economía y Finanzas, Petroecuador, etc.; de donde se obtuvieron los datos del problema planteado.

En esta Investigación las unidades de estudio comprendieron: Las Proformas Anuales y Cuatrianuales del Presupuestos Generales del Estado comprendidas en el período 2009-2018, El Decreto Ejecutivo 619 del 21 de diciembre del 2018, y; la Ley de Hidrocarburos del Ecuador.

Se utilizó el Método deductivo, en el análisis cualitativo, para analizar e interpretar los diversos documentos, y; el Método Inductivo, en el análisis cuantitativo, de las tablas y gráficos estadísticos, presentados por las Instituciones Estatales, mencionadas en las unidades de estudio.

El criterio muestral es no probabilístico discrecional, debido a que el autor escogió la muestra (Los subsidios a los Hidrocarburos en el Ecuador 2009-2018) considerando que al ser más cercanos temporalmente a la fecha de la Investigación tienen mayor representatividad.

La tecnica utilizada es el Análisis de Documentos, y el instrumento, la Ficha de contenido. 


\section{Subsidios a los hidrocarburos en Ecuador}

Vol. 3, núm. 4, (2019)

Carlos Roberto Hernández Miticanoy; Miguel Garcia Jiménez; Miguel Ángel Aizaga Villate

Los indicadores serán las relaciones porcentuales que representa el Monto destinado a subsidiar los derivados de los Hidrocarburos en Ecuador, frente al Monto del Presupuesto General del Estado y algunas de las variables más importantes del Presupuesto General del Estado como son: Ingresos por Impuesto, Ingresos por Tasas y Contribuciones, Ingresos por Financiamiento, Ingresos Petroleros, y; Egresos por Amortización de Deuda, por Inversión en Obra Pública, Monto total de Subsidios y el destinado la Gas Licuado de Petróleo.

Finalmente, la Técnica utilizada en el procesamiento de datos es la Estadística Descriptiva.

\section{Resultados.}

Análisis de la Carga Fiscal que representan los subsidios a los derivados de los Hidrocarburos para el Presupuesto General del Estado según las programaciones Anuales y Cuatrianuales del Ecuador

Tabla 2. Relación Montos de los Subsidios a los Derivados de los Hidrocarburos - Presupuesto General del Estado Período 2009-2018 (millones de dólares)

\begin{tabular}{|c|c|c|c|}
\hline AÑO & PGE & 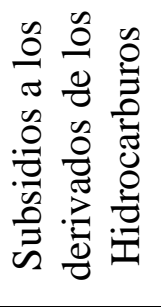 & 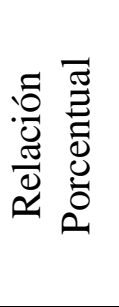 \\
\hline 2009 & 19167,81 & 1573,40 & 8,21 \\
\hline 2010 & 21282,06 & 2641,40 & 12,41 \\
\hline
\end{tabular}


Carlos Roberto Hernández Miticanoy; Miguel Garcia Jiménez; Miguel Ángel Aizaga Villate

\begin{tabular}{|c|c|c|c|}
\hline 2011 & 23950,25 & 2571,09 & 10,74 \\
\hline 2012 & 26109,28 & 2867,02 & 10,98 \\
\hline 2013 & 32366,83 & 4539,64 & 14,03 \\
\hline 2014 & 34300,64 & 3888,65 & 11,34 \\
\hline 2015 & 36317,12 & 3494,84 & 9,62 \\
\hline 2016 & 29835,10 & 1888,10 & 6,33 \\
\hline 2017 & 36818,13 & 1798,57 & 4,89 \\
\hline 2018 & 34853,00 & 1707,04 & 4,90 \\
\hline Total & 295000,21 & 26969,75 & 9,35 \\
\hline
\end{tabular}

Fuente: Ministerio de Economía y Finanzas 2019 (Proformas Aprobadas)

\section{Elaborado por: Carlos Hernández}

Como se puede apreciar, el Monto de los subsidios a los Hidrocarburos durante los diez años, en promedio, tiende al $10 \%$ del Monto total del Presupuesto General del Estado. En el primer quinquenio analizado, los montos superan el promedio, luego, a partir del 2015 empezaron a reducirse gradualmente, coincidiendo con los años en los que el petróleo redujo sustancialmente sus precios internacionales. Como se mencionó en el apartado teórico: "En el período 2010-2014 los mercados internacionales mantuvieron precios de 70 a 100 dólares por barril de petróleo." (Carlosama y Ramos, 2018, p. 68), en cambio a partir del 2015 el precio se redujo hasta llegar a los 20 dólares lo que representaba pérdidas para el estado en un promedio de 6 usd por barril, “Correa señaló que la venta de petróleo, ni siquiera cubre los costos de producción, y que ello le obligará a tomar medidas de ajuste fiscal y la reducción del programa de inversiones" (Diario El Comercio, 2016, p. 1).

Otras razones por las que se han reducido los costos de los subsidios son: La inauguración de las Hidroeléctricas, que a su vez solventaron proyectos como: el uso de las cocinas de inducción y la sustitución de energía eléctrica producida por la quema de combustibles fósiles. También se 


\section{Subsidios a los hidrocarburos en Ecuador}

Vol. 3, núm. 4, (2019)

Carlos Roberto Hernández Miticanoy; Miguel Garcia Jiménez; Miguel Ángel Aizaga Villate

observa que el Montos asignado a subsidiar los derivados de los hidrocarburos, no responden a ningún patrón de crecimiento, pues un año crece y al siguiente decrece, además se esperaría una significativa reducción del Monto destinado a subsidiar el consumo de los derivados de los hidrocarburos, a partir de la promulgación del decreto ejecutivo $\mathrm{N}^{\circ} 619$, que modificó los precios, congelados desde el 2005. Todo lo contrario, para el año 2019, se presenta un valor que desentona con las reducciones a los subsidios de los Hidrocarburos realizados en los últimos tres años, 4176,46 millones de dólares.

Esta cifra (4176,46 millones de dólares), aunque pertenece a una programación que no está dentro del período de análisis, deja ver que existen variables fuera de los objetivos de este estudio, que influyen considerablemente en el crecimiento del costo anual de los subsidios a los derivados de los Hidrocarburos en Ecuador.

Los subsidios a los derivados de los Hidrocarburos alcanzan un Monto acumulado de 26969,75 millones de dólares en la década estudiada, sin duda representan un costo de oportunidad altísimo para el país. Si este Monto se compara con los costos de la construcción de la Refinería del Pacífico (15000 millones de dólares), proyecto que pretendió lograr el gobierno de esa década. Cabe la suposición de que, con ese Monto, se pudieron construir casi dos refinerías, al costo propuesto inicialmente, y; tres, si se hubiera construido según los recientes estudios presentados por el gobierno de Lénin Moreno.

La construcción de la Refinería del Pacifico podría tener un valor inferior al previsto por el Gobierno anterior. Un informe de la firma francesa Lazard determinó que 
el costo estimado de esta infraestructura estaría entre los USD 8000 y 10000 millones. (El Comercio, 2017, p.1).

Este es solo un ejemplo de inversiones que se pudieron realizar, otras son: los proyectos Hidroeléctricos, los Puertos, la ampliación de la Corporación Nacional de Telecomunicaciones EP (CNT), Yachay, Infraestructura Agropecuaria, etc. Y pensando en el futuro, inversiones en investigaciones e infraestructura para aprovechar nuevas fuentes de energía, como la eólica, solar, y otras.

Análisis Comparativo de los Montos de los principales Ingresos del Presupuesto General del Estado y los de los Subsidios a los Derivados de los Hidrocarburos

Imagen 1. Relación Montos de los Principales Ingresos del Presupuesto General del Estado Subsidios a los derivados de los Hidrocarburos. Periodo (2009-2018) (Millones de Dólares) 


\section{Subsidios a los hidrocarburos en Ecuador}

Vol. 3, núm. 4, (2019)

Carlos Roberto Hernández Miticanoy; Miguel Garcia Jiménez; Miguel Ángel Aizaga Villate

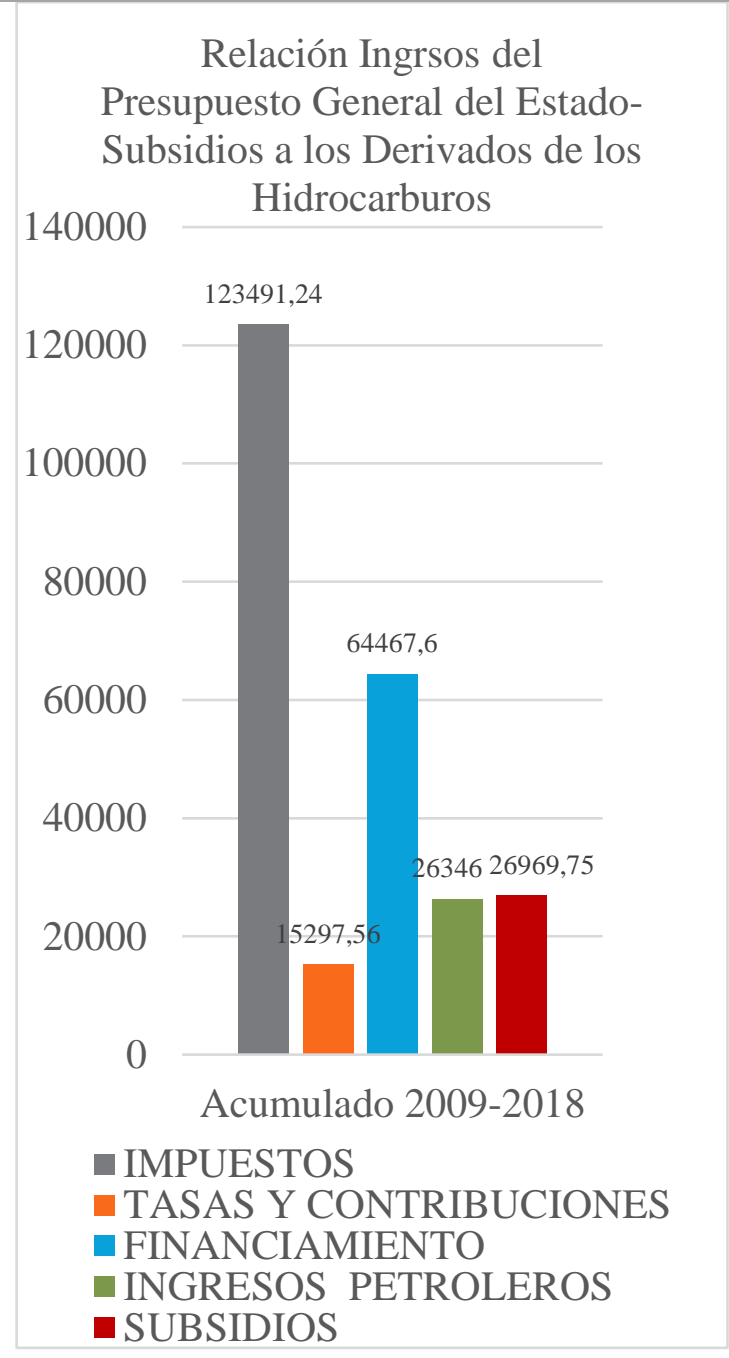

Fuente: Ministerio de Economía y Finanzas 2019 (Proformas Aprobadas)

Elaborado por: Carlos Hernández

Al observar el gráfico 1, lo que más llama la atención es que El Monto de los subsidios a los Derivados de los Hidrocarburos comprende el $42 \%$ del Monto de Financiamiento. Partiendo de aquello, al colocarse en la situación hipotética de que el Ecuador no fuera productor de Petróleo, ¿Qué objetivos perseguiría al endeudarse año tras año, y destinar el $42 \%$ de esa deuda, a beneficio de los sectores más pudientes del país, a fomentar el contrabando y la contaminación ambiental? 
Subsidios a los hidrocarburos en Ecuador

Vol. 3, núm. 4, (2019)

Carlos Roberto Hernández Miticanoy; Miguel Garcia Jiménez; Miguel Ángel Aizaga Villate

A sabiendas de que "Reducir o eliminar los subsidios directos y pos impuesto a los combustibles tendría efectos positivos sobre el medioambiente, la economía, el equilibrio fiscal, e incluso, la salud.” (Fierro, 2014, p.2). Y además contradiciendo a lo estipulado en La Constitución 2008, artículo 290, literal 3: “Con endeudamiento público se financiarán exclusivamente programas y proyectos de inversión para infraestructura, o que tengan capacidad financiera de pago" (Asamblea Constituyente, 2008, p. 141).

Haciendo una comparación del Monto que representan los subsidios a los derivados de los Hidrocarburos, con respecto del Monto por Impuestos (22\%), luego comparado al Monto de Tasas y Contribuciones, vemos que lo supera en un $76 \%$. Se podría decir entonces que los subsidios constituyen un perjuicio para el Estado y para la población más vulnerable, pues ayudan a mantener un sistema que redistribuye mal la riqueza, devolviendo los impuestos a quienes no lo necesitan.

El efecto distributivo es muy regresivo, pues la mayor parte del beneficio de los subsidios “va a los quintiles superiores en la distribución del ingreso. En promedio, en los países en desarrollo, el quintil de ingresos más altos captura $43 \%$ del subsidio, mientras el quintil de menores ingresos captura apenas 7\% del subsidio." (Fierro, 2014, p.2).

Los Montos de Ingresos por venta de Petróleo y Subsidios a sus derivados, son técnicamente iguales, esto hace suponer que el $50 \%$ de la producción petrolera no se invierte, se gasta. Se regala a quien más dinero tiene. Suponiendo que el otro $50 \%$ estuviera bien invertido, cosa que no se cumple, al no existir una política clara o un fin específico, del uso de los recursos del sector petrolero y sus derivados. 


\section{Subsidios a los hidrocarburos en Ecuador}

Vol. 3, núm. 4, (2019)

Carlos Roberto Hernández Miticanoy; Miguel Garcia Jiménez; Miguel Ángel Aizaga Villate

Análisis Comparativo de los Montos Principales Egresos - Subsidios a los derivados de los

Hidrocarburos en el Presupuesto General del Estado

Imagen 2. Relación Montos de Principales Egresos del Presupuesto General del EstadoSubsidios a los derivados de los Hidrocarburos Periodo 2009-2018 (Millones de Dólares

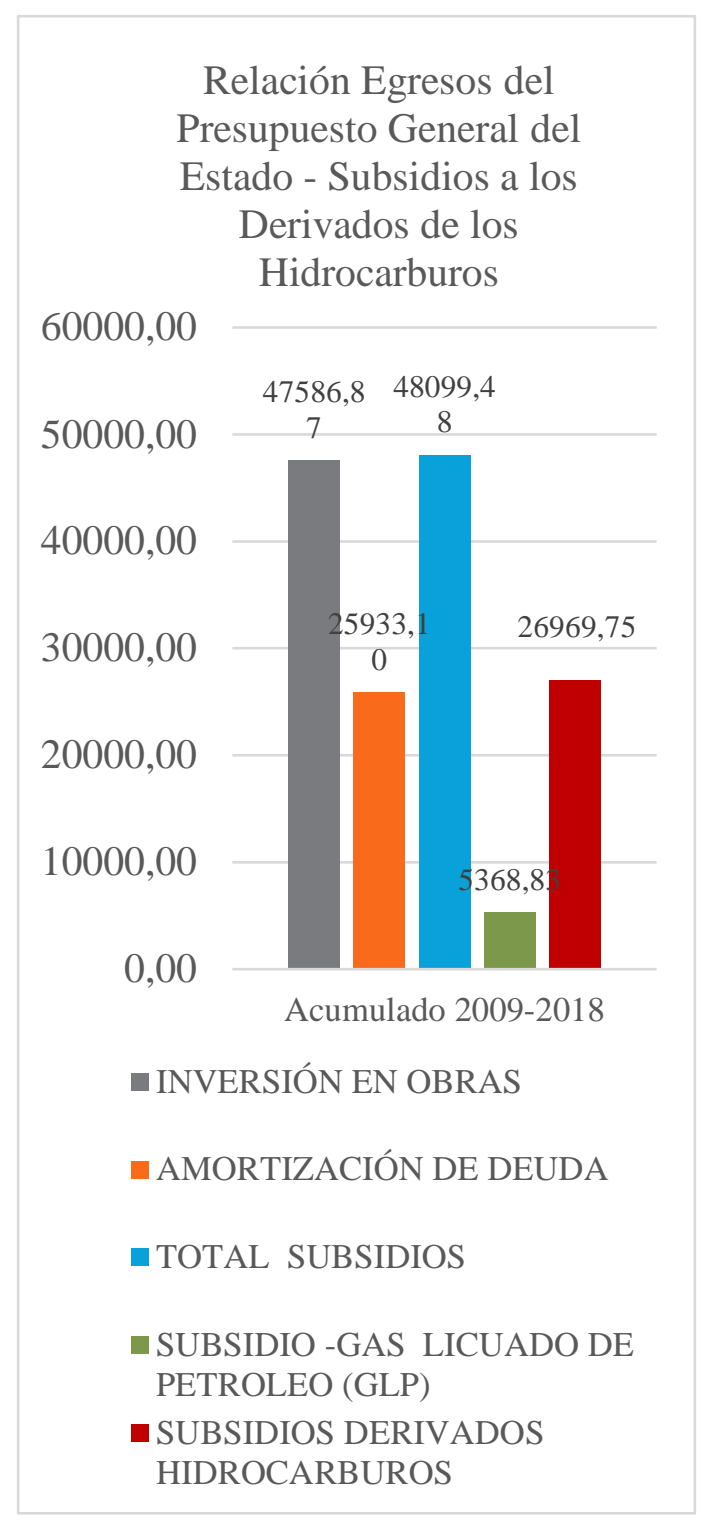

Fuente: Ministerio de Economía y Finanzas 2019 (Proformas Aprobadas) 


\section{Elaborado por: Carlos Hernández}

El Monto acumulado de los Subsidios a los derivados de los Hidrocarburos durante la década en estudio, supera en 1\% al Monto de la Amortización de Deuda. Al comparar este Monto, con el de la Deuda Pública con corte al 31 de diciembre 2018, (49429,2 millones de dólares), se puede concluir que, de haberse destinado el Monto de los Subsidios a los derivados de los Hidrocarburos, para pagar la deuda; el Ecuador contaría actualmente con una deuda de 23000 millones de dólares, aproximadamente. Monto que técnicamente, sería fácil de manejar.

En cuanto a la Inversión en Obra Pública, el Monto destinado al subsidio de los Hidrocarburos representa un 57\% de este, enfatizando que en el período en estudio, hubo mayor Inversión que en muchos gobiernos anteriores, y supera tambien al de estos dos últimos años.(2017-2018).Sin embargo de aquello, se pudo y se podría en el futuro, hacer mucho más.

Castaño (2003) asevera que las inversiones, a más de generar fuentes de trabajo, producen moviento comercial, de capital, de ahorro, producen ganancias que pueden cubrir gastos o nuevas inversiones. (efecto multiplicador). Pero del mismo modo la disminución de las inversiones da lugar a una contracción multiplicada de ingresos. Por lo que la depresión o expansión puede ser provocada por el atesoramiento o desatesoramiento de la comunidad, los empresarios o las políticas del estado intervencionista. ¿Cuál realidad vive el Ecuador? Ciertamente la que provoca el segundo panorama, donde el gasto de consumo va rebajando año tras año. Por consiguiente, es en este punto donde se puede palpar con mayor claridad, el costo de oportunidad que ha pagado y seguirá pagando el Ecuador, de no modificar radicalmente sus políticas en el campo de los Hidrocarburos. 


\section{Subsidios a los hidrocarburos en Ecuador}

Vol. 3, núm. 4, (2019)

Carlos Roberto Hernández Miticanoy; Miguel Garcia Jiménez; Miguel Ángel Aizaga Villate

Finalmente, al comparar el Monto de los subsidios a los derivados de los Hidrocarburos con el Monto destinado al Total de los subsidios, supera a la suma de todos los otros (56\%). Al observar aquel destinado al Subsidio del Gas licuado de petróleo, se comprueba que representa el $11 \%$ del total de los subsidios y el 20\% de los Subsidios a los derivados del petróleo. Al comparar el Monto total del Presupuesto General del Estado en los diez años estudiados, se observa que el subsidio al gas licuado de petróleo, le equivale al 1,8\%. Este Monto es manejable para transferirlo a todos los beneficiarios del Bono de Desarrollo Social, como una solución para que no afecte negativamente a los sectores menos favorecidos, la decisión de eliminar los subsidios a derivados de los Hidrocarburos.

Examinar la Normativa y Políticas Públicas actuales, para reconocer el campo legal de la Administración de los subsidios a los Hidrocarburos en el Ecuador

De los documentos analizados se encontró una Ley de Hidrocarburos aprobada en 1978, la cual fue reformada parcialmente en varias ocasiones, la última vez, el 21 de mayo de 2018. A pesar de aquello, tiene seis artículos para contrataciones: (25, 45, 46, 47, 48 y 53), con precios expresados en sucres, moneda no vigente en el país desde el 2000. Surge entonces la necesidad de reformar o diseñar otra Ley, acorde a las necesidades actuales.

Se debe señalar que la Constitución (2008) en el Titulo VI Régimen de Desarrollo, nos da las normativas necesarias para Administrar de manera adecuada, el petróleo y todos los recursos que posee el estado, a fin de que el desarrollo del país sea sostenible, justo y equitativo.

Desde el dos mil siete el estado recuperó el papel protagónico en el sector petrolero, lo cual ha incrementado los ingresos al Presupuesto General del Estado. A finales del 2018, mediante 
Subsidios a los hidrocarburos en Ecuador

Vol. 3, núm. 4, (2019)

Carlos Roberto Hernández Miticanoy; Miguel Garcia Jiménez; Miguel Ángel Aizaga Villate

decreto ejecutivo 619, se descongeló los precios de los derivados de los Hidrocarburos. Se esperaría que estas reformas contribuyan a disminuir los valores destinados a los subsidios de los derivados de los Hidrocarburos, no obstante, el valor presupuestado para el 2019, alcanza un Monto de 4176,47 millones de dólares, contradiciendo a las aspiraciones de las reformas.

Para concluir, haremos referencia a la Regla fiscal, la cual refiere lo siguiente:

Art. 81.- Para garantizar la conducción de las finanzas públicas de manera sostenible, responsable, transparente y procurar la estabilidad económica; los egresos permanentes se financiarán única y exclusivamente con ingresos permanentes. No obstante, los ingresos permanentes pueden también financiar egresos no permanentes. Los egresos permanentes se podrán financiar con ingresos no permanentes en las situaciones excepcionales que prevé la Constitución de la República, para salud, educación y justicia; previa calificación de la situación excepcional, realizada por la Presidenta o el Presidente de la República. (Asamblea Nacional, 2010, p. 29).

La pregunta imprescindible, ¿Los recursos económicos provenientes de la actividad petrolera son permanentes o no permanentes, y los subsidios a sus derivados pertenecen a gastos permanentes o no permanentes?

\section{Discusión}

Los Montos destinados a subsidiar el consumo de derivados de los Hidrocarburos en Ecuador, durante el período 2009-2018, analizados longitudinalmente, no cumplen ningún patrón de crecimiento y pueden variar drásticamente de una programación a otra, como ocurre en los años, 2012-2013 y luego en los años, 2015-2016, no obstante, en ningún momento han sido 


\section{Subsidios a los hidrocarburos en Ecuador}

Vol. 3, núm. 4, (2019)

Carlos Roberto Hernández Miticanoy; Miguel Garcia Jiménez; Miguel Ángel Aizaga Villate

inferiores al 5\% del Presupuesto General del Estado, y en años como los comprendidos en el período 2010- 2014, esos Montos, superan el 10\% del Presupuesto General del Estado. Finalmente, el promedio porcentual en el período 2009-2018 es de 35\%. También se puede apreciar transversalmente que el Monto acumulados (2009-2018) de los Subsidios a los derivados de los Hidrocarburos, equivale al 9,2 \% del Monto acumulado del Presupuesto General del Estado.

El Monto acumulado de los Subsidios a los derivados de los Hidrocarburos alcanza una cifra de 26969,75 millones de dólares, al ser comparada con el Monto de los Ingresos petroleros prácticamente tiene el mismo valor (ver gráfico 1), se concluye que el 50\% de las ganancias de la actividad petrolera no se está invirtiendo como se debería, considerando que el petróleo es un recurso no renovable, con pocos años de explotación futura. Confirma también lo expuesto por varios autores citados, "los subsidios se los utiliza como dadiva para mantener contento al pueblo y seguro al gobernante en el cargo, así como para garantizar el consumo de este recurso"

El punto crítico, surge al comparar el Monto acumulado de los Subsidios a los derivados de los Hidrocarburos, con el Monto correspondiente al Ingreso por Financiamiento, pues fácilmente se puede concluir que, el Ecuador destina el $42 \%$ de su deuda, a financiar subsidios ineficaces y desenfocados, lo que le provoca un estancamiento económico, pérdida de credibilidad crediticia, injusta distribución y redistribución de riqueza y desinversión. En consecuencia, una pérdida permanente de plazas de empleo y calidad de vida de su población.

Si se compara con los Montos de Pago de Deuda, Inversión en Obra Pública, El Total de Subsidios y el subsidio al Gas Licuado de Petróleo, (ver gráfico 2), al cual técnicamente lo contiene cinco veces, se llega a la conclusión de que, con los Montos anuales de los Subsidios a los 
Subsidios a los hidrocarburos en Ecuador

Vol. 3, núm. 4, (2019)

Carlos Roberto Hernández Miticanoy; Miguel Garcia Jiménez; Miguel Ángel Aizaga Villate

derivados de los Hidrocarburos, se pagaría con facilidad la deuda pública, en una década, dado que para la planificación del 2019 otra vez aparece un Monto muy alto, 4176,47 millones de dólares destinado a subsidiar el uso de derivados de Hidrocarburos, (13\%) del Presupuesto General del Estado (Ministerio de Econimía y Finanzas, 2018, p. 35).

En cuanto a la Ley de Hidrocarburos, esta se encuentra desactualizada, con necesidades de varios cambios, acordes a las necesidades de la época actual y con visión futurista.

En el artículo base para este apartado, La Política Publica de Hidrocarburos en el Ecuador Periodo 2007-2015: Hacia la Seguridad Energética (2018), se deja claro que en este período el estado recuperó el papel protagónico en el sector, al aplicar las siguientes políticas: “a) Incrementar la contribución del sector petrolero al desarrollo nacional, b) La seguridad y soberanía energética, c) La eficiencia institucional del sector, d) En el consumo de hidrocarburos, y; e) Disminuir el impacto social y ambiental de las actividades petroleras." (Cevallos, 2018, p. 26). Estas políticas deberían haber beneficiado en algún campo o en cierto modo, incrementando los ingresos al Presupuesto General del Estado, no obstante, concluye que, las Políticas no contribuyeron con el incremento de la seguridad energética. (Cevallos, 2018).

\section{Conclusiones.}

El Monto destinado a subsidiar el uso de los derivados de los Hidrocarburos en el Ecuador durante el período 2009 - 2018, representó el 9,35 \% del Presupuesto General del Estado. Esto originó un alto costo de oportunidad para el Estado, evidenciado en la Desinversión, Crecimiento de la deuda Pública, Falta de Liquidez para Amortizar la misma Deuda y finalmente una ineficaz Distribución y Redistribución de la Riqueza. 


\section{Subsidios a los hidrocarburos en Ecuador}

Vol. 3, núm. 4, (2019)

Carlos Roberto Hernández Miticanoy; Miguel Garcia Jiménez; Miguel Ángel Aizaga Villate

El Monto asignado a subsidiar el consumo de los derivados de los Hidrocarburos en el Ecuador, es superado solamente por el Monto total de los Impuestos (22\%) y el de Financiamiento Público (42\%), a los demás los equipara o los supera como al monto de Tasas y Contribuciones. Evidenciando una ineficaz redistribución de la riqueza y un endeudamiento para causas incostitucionales e injustas.

El Monto asignado a subsidiar el consumo de los derivados de los Hidrocarburos en el Ecuador, evidencia que se ha dejado de pagar la Dueda y dejado de Invertir, para mantener un subsidio ineficaz.

El Ecuador cuenta con una excelente Constitución, que dedica un apartado completo al Desarrollo Sostenible, lamentablemente, no se cumple en la práctica. Por otra parte, la Ley de Hidrocarburos necesita reformas o en su defecto, es necesaria la creación de una nueva Ley.

Se recomienda la eliminación urgente de todos los subsidios a los derivados de los Hidrocarburos, a fin de generar mayores Inversiones en Sectores Estratégicos, Investigación y Sectores Sociales, como: Educación y Salud.

Poner en práctica lo que manda la Constitución 2008.

Constituir una nueva Ley de Hidrocarburos, coherente con las necesidades económicas del país, aprovechando experiencias de paises vecinos, que han eliminado los subsidios a los derivados de los Hidrocarburos y aprovechan otras fuentes de energia, con buenos resultados.

\section{Bibliografía.}

Asamblea Constituyente. (2008). Constitución 2008. Montecristi: Asamblea Nacional. 
Subsidios a los hidrocarburos en Ecuador

Vol. 3, núm. 4, (2019)

Carlos Roberto Hernández Miticanoy; Miguel Garcia Jiménez; Miguel Ángel Aizaga Villate

Carlosama, W., \& Ramos, K. (2018). Análisis del Financiamiento del Gasto Público del Presupuesto General del Estado. Sangolquí: ESPE.

Castaño, R. (2003). Ideas Económicas Mínimas. Bogotá: Ecoe Ediciones.

CEPAL. (2006). Gobernabilidad Fiscal en Ecuador. Santiago de Chile: Naciones Unidas.

Cevallos, M. C. (2018). La Política Pública de Hidrocarburos en el Ecuador, Período 2007-2015: Hacia la Seguridad Energética. Quito: Instituto de Altos Estudios Nacionales.

Consejo Supremo de Gobierno. (15 de noviembre de 1978). Ley de Hidrocarburos, 1978. Quito, Pichincha, Ecuador: Registro Oficial. Recuperado de http://www.ambiente.gob.ec/wpcontent/uploads/downloads/2018/05/Ley-de-Hidrocarburos-1978.pdf

Cueva, S., \& Maria, O. (2013). Ingresos Fiscales por Explotación de Hidrocarburos en Ecuador. Quito: Banco Interamericano de Desarrollo.

Diario El Comercio. (7 de Marzo de 2016). ¿Ecuador está preparado para un petróleo a un precio de USD 20?: El comercio.com. Recuperado de ElComercio.com: https://www.elcomercio.com/actualidad/ecuador-preparados-petroleo-preciopetroecuador.html.

Diario El Comercio. (06 de 07 de 2017). www.elcomercio.com. Recuperado de https://www.elcomercio.com/actualidad/negocios-costo-refineria-pacifico-oscilacion.html

El Teelegrafo. (16 de Octubre de 2018). Sector automotor espera cerrar 2018 con un crecimiento del 33\%: eltlegrafo.com. Recuperado el 04 de 03 de 2019, de www.eltlegrafo.com.ecnoticias:

https://www.eltelegrafo.com.ec/noticias/economia/4/sectorautomotriz-2018-crecimiento33

El Universo. (15 de Enero de 2019). Ecuador cumplió diez años con déficit en presupuestos del Estado:eluniverso. Recuperado el 04 de 03 de 2019, de www.eluniverso.com: https://www.eluniverso.com/noticias/2019/01/15/nota/7139165/decada-deficitpresupuestos-estado

Espinoza, S., \& Guayanlema, V. (2017). Balance y proyecciones del sistema de subsidios energéticos en Ecuador. Quito: Friedrich-Ebert-Stiftung (FES-ILDIS) Ecuador.

Fierro, L. (22 de Noviembre de 2014). El Ecuador tiene el mayor nivel de subsidios a los combustibles: drive google. com. Recuperado de drive google. com: https://drive.google.com/file/d/0B2keH2NAJ6iON3FLWm9oQ0taTDQ/view

Mendoza, M. (2014). Panorama Preliminar de los Subsidios y los impuestos a las gasolinas y diésel en los paises de América Latina. Santiago de Chile: CEPAL. 


\section{Subsidios a los hidrocarburos en Ecuador}

Vol. 3, núm. 4, (2019)

Carlos Roberto Hernández Miticanoy; Miguel Garcia Jiménez; Miguel Ángel Aizaga Villate

Ministerio de Econimía y Finanzas. (20 de Junio de 2019). Proformas Aprobadas: Ministerio de Economia $y$ Finanzas. Recuperado de www.finanzas.gob.ec: https://www.finanzas.gob.ec/proformas-aprobadas/

Ministerio de Economía y Finanzas. (14 de 06 de 2019). Deuda Pública: www.finanzas.gob.ec. Recuperado de www.finanzas.gob.ec: https://www.finanzas.gob.ec/deuda-publica/

Moreno, L. (21 de Diciembre de 2018). Decreto Ejecutivo 619. Reforma al Decreto Ejecutivo 338. Quito, Pichincha, Ecuador: Registro Oficial.

Petroecuador, EP. (2018). Informe Estadístico 1972-2017. Quito: Petroecuador

Universidad Autónoma del Estado de Hidalgo. (12 de 03 de 2019). Importancia de los Hidrocarburos:www.uaeh.edu.mx. recuperado de www.uaeh.edu.mxn Web site: https://www.uaeh.edu.mx/scige/boletin/prepa3/n2/m11.html.

Xataka. (3 de Enero de 2018). China se sigue esforzando por ser un país verde; Xataka. Recuperado de Xataka Web site: https://www.xataka.com

$$
\text { (9) (1) }(9)
$$

RECONOCIMIENTO-NOCOMERCIAL-COMPARTIRIGUAL

CC BY-NC-SA

ESTA LICENCIA PERMITE A OTROS ENTREMEZCLAR, AJUSTAR Y CONSTRUIR A PARTIR DE SU OBRA CON FINES NO COMERCIALES, SIEMPRE Y CUANDO LE RECONOZCAN LA AUTORÍA Y SUS NUEVAS CREACIONES ESTÉN BAJO UNA LICENCIA CON LOS MISMOS TÉRMINOS. 